\title{
A Bayesian Approach for on-Line Max and Min Auditing
}

\author{
Gerardo Canfora \\ RCOST - Department of Engineering \\ University of Sannio \\ gerardo.canfora@unisannio.it
}

\author{
Bice Cavallo \\ RCOST - Department of Engineering \\ University of Sannio \\ bice.cavallo@unisannio.it
}

\begin{abstract}
In this paper we consider the on-line max and min query auditing problem: given a private association between fields in a data set, a sequence of max and min queries that have already been posed about the data, their corresponding answers and a new query, deny the answer if a private information is inferred or give the true answer otherwise. We give a probabilistic definition of privacy and demonstrate that max and min queries, without "no duplicates" assumption, can be audited by means of a Bayesian network. Moreover, we show how our auditing approach is able to manage user prior-knowledge.
\end{abstract}

\section{Keywords}

Bayesian network, Statistical database, Auditing

\section{INTRODUCTION}

The protection of privacy in statistical database (SDB) has been a problem of growing concern in recent years [5]. The goal is to provide statistical information about groups of individuals while protecting their privacy; only statistical information must be available and no sequence of queries should provide protected information about the individuals. If an user is able to infer protected information, then the SDB is compromised.

A number of disclosure control methods to protect a SDB have been proposed in the literature (see [1] for a survey); one of these is auditing [3], [4], [8], [9], [10], [14]. Two kinds of auditing have been studied:

1. On-line auditing. The queries are answered one by one in sequence and the auditor has to determine whether the SDB is compromised when answering a new query;

2. Off-line auditing. Given a set of queries $\left\{q_{1}, \ldots, q_{t}\right\}$, the auditor has to identify a maximum subset of queries that can be answered simultaneously without compromising the SDB.
In this paper we consider the first case, in particular the on-line max and min query auditing over real-valued data. Consider for example, a company database containing salaries of employees. A user may want to determine the max or a min salary of the employees in a subset of records in the database. She cannot, however, be allowed to glean the salary of any one employee in particular.

In an on-line max and min auditing problem, given a set of max and min queries $\left\{q_{1}, q_{2}, \ldots q_{t-1}\right\}$, the corresponding answers $\left\{m_{1}, m_{2}, \ldots m_{t-1}\right\}$ and the current query $q_{t}$, the auditor denies the answer to $q_{t}$ if and only if a privacy breach occurs.

In [11] the authors deal with the max and min auditing, but the combinations of max and min queries in presence of duplicated sensitive values remains an open problem.

The original contribution of this paper is threefold:

1. to extend the classical notion of privacy to a probabilistic notion;

2. to show as to deal with the on-line max and min auditing, without "no duplicates" assumption, by means of a Bayesian network;

3. to manage user prior-knowledge in addition to the past history of user queries.

The rest of the paper is organized as follows: section 2 places our work in the context of previous research; section 3 presents a probabilistic approach for on-line max auditing; section 4 introduces the Bayesian network as a graphical model to deal with uncertainty and shows how a Bayesian network is able to deal with the on-line max and min auditing problem; section 5 outlines how our model deals with prior-knowledge; section 6 discusses conclusions and future research.

\section{BACKGROUND}

In this section we provide the notation that we will use in the sequel and place our work in the context of previous research.

\subsection{Notation}

We assume that:

- $T$ is a table with $n$ records; 
- $K=\{1,2, \ldots, n\}$;

- $X$ and $Y$ are two fields of $T$ such that the elements of $X$ represented by $x_{i}$ are distinct among them (each $x_{i}$ identifies uniquely a subject) and the elements of $Y$, represented by $y_{i}$, are real numbers;

- the sensitive field $Y$ has $r$ distinct values $(r \leq n)$;

- the private information takes the form of an association, $\left(x_{i}, y_{i}\right) \subseteq X \times Y$, that is the pair of values in the same tuple;

- $S=\left\{s_{1}, \ldots, s_{r}\right\}$ is the set of distinct values of $Y$ with $s_{i}<s_{i+1}, \forall i \in\{1, \ldots, r-1\}$;

- $S_{0}=\left\{s_{0}, s_{1}, \ldots, s_{r}, s_{r+1}\right\}$, with $s_{i} \in S, \forall i \in\{1, \ldots, r\}$, $s_{0}$ a real number such that $s_{0}<s_{1}$ and $s_{r+1}$ a real number such that $s_{r+1}>s_{r}$;

- a $l$-query $q$ is a subset of $K$, that is $q=\left\{i_{1}, \ldots i_{l}\right\} \subseteq K$. Let us assume that $i_{j}<i_{j+1} \quad \forall j \in\{1, \ldots, l-1\}$; it is not restrictive, for example $q_{1}=\{1,2\}$ and $q_{2}=\{2,1\}$ represent the same query. In this paper, we use the terms query and l-query interchangeably;

- the answer corresponding to a max query $q$ is $m=$ $\max \left\{y_{i_{j}} \mid i_{j} \in q\right\}$

- the answer corresponding to a min query $q$ is $m=$ $\min \left\{y_{i_{j}} \mid i_{j} \in q\right\}$

- $l=|q|>1$, because if $q=\{j\}$, clearly, $y_{j}$ is breached irrespective of the value of $m$ and the association $\left(x_{j}, m\right)$ is disclosed.

\subsection{Related work}

Given a table $T$, the private associations $\left(x_{j}, y_{j}\right)$, a set of max and min queries $\left\{q_{1}, \ldots, q_{t}\right\}$, and the set of the corresponding answers $\left\{m_{1}, \ldots, m_{t}\right\}$, in [9] and in [11] the authors define, for each element $y_{j}$, the upper bound $\mu_{j}$ as follows:

DeFinition 1. $\forall y_{j}, \mu_{j}=\min \left\{m_{k} \mid j \in q_{k}\right.$ with $q_{k}$ a max query\} is the minimum over the answers to the max queries containing $j$.

In other words, $\mu_{j}$ is the best possible upper bound for $y_{j}$ that can be obtained from the answers to the max queries.

Similarly, the lower bound $\lambda_{j}$ is defined as follows:

Definition 2. $\forall y_{j}, \lambda_{j}=\max \left\{m_{k} \mid j \in q_{k}\right.$ with $q_{k}$ a min query $\}$ is the maximum over the answers to the min queries containing $j$.

Moreover, if $q_{k}$ is a max query, then an extreme element for $q_{k}$ is define as follows:

DEFINITION 3. $j$ is an extreme element for the query $q_{k}$ if $j \in q_{k}$ and $\mu_{j}=m_{k}$.

Instead, if $q_{k}$ is a min query, then an extreme element for $q_{k}$ is define as follows:
DEFINITION 4. $j$ is an extreme element for the query $q_{k}$ if $j \in q_{k}$ and $\lambda_{j}=m_{k}$.

In [9], the authors demonstrate, for the on-line max auditing, the following theorem:

TheOrem 1. A value $y_{j}$ is uniquely determined if and only if there exists a query $q_{k}$ for which $j$ is the only extreme element.

This means that the private association $\left(x_{j}, y_{j}\right)$ is disclosed; this theorem is valid also for the on-line min auditing.

EXAMPLE 1. Let $q_{1}=\{2,3,4\}$ a max query. If the user submits the max query $q_{2}=\{1,2,3,4,5\}$ and $m_{1}<m_{2}$, then the auditor can answer, because $q_{1}$ has 3 extreme elements, that are 2, 3, 4 and $q_{2}$ has 2 extreme elements, that are 1 and 5 .

EXAMPLE 2. Let $q_{1}=\{1,2,3,4\}$ a max query. If the user submits the max query $q_{2}=\{1,2,3,4,5\}$ and $m_{1}<m_{2}$, then the auditor must deny the answer to $q_{2}$, because $q_{1}$ has 4 extreme elements, that are 1, 2, 3, 4 and $q_{2}$ has only an extreme element, that is 5 .

EXAMPLE 3. Let $q_{1}=\{1,2,3\}$ a min query. If the user submits the min query $q_{2}=\{1,4\}$ and $m_{1}>m_{2}$, then the auditor must deny the answer to $q_{2}$, because $q_{1}$ has 3 extreme elements, that are 1, 2, 3 and $q_{2}$ has only an extreme element, that is 4 .

Focusing on max auditing, reference [8] proposes an enhanced approach that considers the implicit delivery of information that derives from denying the answer to a query.

In [11] the authors deal with on-line max and min auditing with "no duplicates"assumption. They provide the following theorem:

TheOREM 2. Given a set of queries, $\left\{q_{1}, \ldots, q_{t}\right\}$ and the corresponding answers $\left\{m_{1}, \ldots, m_{t}\right\}$, the database is secure if and only if every max query or min query has more than one extreme element and does not exist any max query, $q_{i}$, and min query $q_{j}$ such that $m_{i}=m_{j}$.

EXAMPLE 4. Let $q_{1}=\{2,3\}$ a max query. If the user submits the min query $q_{2}=\{1,3\}$ and $m=m_{1}=m_{2}$, then the auditor must deny the answer to $q_{2}$, because the user discloses the association $\left(x_{3}, m\right)$.

The question of auditing combination of max and min queries in presence of duplicated remains, for the authors, an open problem, because, in their approach, the duplicates make the problem harder. To see why duplicated make the problem harder, we consider the following example: 
EXAMPLE 5. Let $q_{1}=\{3,5\}$ a max query and $m_{1}=98$, $q_{2}=\{4,6\}$ a max query and $m_{2}=98$. Now, if the auditor gives the answer to min query $q_{3}=\{5,6\}$ with $m_{3}=96$, then in addition to the previous information, the user also knows that $\max \left\{y_{3}, y_{4}\right\}$ must be 98 since one of $y_{5}$ or $y_{6}$ has to be 96. Thus, in addition to examining extreme elements for the query that have actually been posed, the auditor needs to examine extreme elements for such inferred queries as well, and there can be a blow up in the number of queries that need to be maintained. This does not happen in the absence of duplicates, since the first two queries could never have the same answer.

\subsection{Problem statement}

We consider the following definition of probabilistic compromise:

Definition 5. A privacy breach occurs if and only if a private association is disclosed with probability greater than a given tolerance probability tol. If a private association is disclosed with tol $=1$, then the $S D B$ is full compromised.

In order to show how the probabilistic definition is more secure than the classical one, we give the following example:

EXAMPLE 6. Given $y_{1}=100$ and $q_{i}, i \in\{1, \ldots, 6\}$ a $\max$ query, with $q_{1}=\{1,2,3,4,5,6,7,8,9\}$ and $m_{1}=100, q_{2}=$ $\{2,3,4\}$ and $m_{2}=99, q_{3}=\{5,6,7,13\}$ and $m_{3}=96$, $q_{4}=\{1,10\}$ and $m_{4}=100, q_{5}=\{1,11\}$ and $m_{5}=100$, $q_{6}=\{7,8\}$ and $m_{6}=94$. If the auditor answers the sequence of queries $\left\{q_{1}, q_{2}, q_{3}, q_{4}, q_{5}, q_{6}\right\}$, then the user knows that $y_{1}=100$ with probability equal to 0.8889 . Even if in classical definition of privacy, a breach does not occur, because each query has at least two extreme elements, if we select tol $=0.87$ then the auditor must deny the answer $m_{6}$ because the private association $\left(x_{1}, 100\right)$ is disclosed with probability equal to 0.8889 . In section 4.2, we will show as our model is able to compute this probability.

With our model, we will be able to answer the following questions:

- What is the probability that an element $y_{j}$ is equal to $\mu_{j}$ after a sequence of queries?

- What is the probability that an element $y_{j}$ is equal to $\lambda_{j}$ after a sequence of queries?

- What are the probabilistic dependencies among the sensitive values after a sequence of queries?

- How we can deal with the blow up in the number of queries that need to be maintained in an on-line max and min auditing? For example, how to represent that $\max \left\{y_{3}, y_{4}\right\}$ must be 98 in the example 5, without maintaining the additional query?

- How we can model the user knowledge after a sequence of queries?

- How we can add user prior-knowledge? For example, what happens, in example 6, if the user knows that, with probability equal to $0.8, y_{1} \geq 100$ ?

\section{A PROBABILISTIC APPROACH}

In this section we deal with max queries; the min case is analogous.

Let $q=\left\{i_{1}, \ldots, i_{l}\right\}$ a $l$-query and $m=\max \left\{y_{i_{1}}, \ldots, y_{i_{l}}\right\}$ the corresponding answer, if the auditor gives the answer $m$ then the user knows that:

$$
\begin{array}{r}
y_{i_{j}} \leq m \quad \forall i_{j} \in q \\
\operatorname{Pr}\left(O R\left(y_{i_{1}}, \ldots, y_{i_{l}}\right)=m\right)=1
\end{array}
$$

The relation in (2) ensures that $\exists j \in\{1, \ldots, l\}$ such that $y_{i_{j}}=m$.

EXAMPLE 7. Let $q=\{1,3\}$ and $m=\max \left\{y_{1}, y_{3}\right\}=8$, then the user knows that it is verified one of the following cases: $y_{1}=8$ and $y_{3}=8 ; y_{1}<8$ and $y_{3}=8 ; y_{1}=8$ and $y_{3}<8$. Since it is likely that the user has not prior knowledge on the domain of the sensitive field $Y$, we assume that each of three cases is equally probable.

Then

$$
\begin{array}{ll}
\operatorname{Pr}\left(y_{i}=8 \mid m=8\right)=\frac{2}{3}, & \forall i \in\{1,3\} \\
\operatorname{Pr}\left(y_{i}<8 \mid m=8\right)=\frac{1}{3}, & \forall i \in\{1,3\}
\end{array}
$$

Moreover

$$
\begin{aligned}
& \operatorname{Pr}\left(y_{1}=8 \mid m=8, y_{3}=8\right)=\frac{1}{2} \\
& \operatorname{Pr}\left(y_{1}<8 \mid m=8, y_{3}=8\right)=\frac{1}{2} \\
& \operatorname{Pr}\left(y_{3}=8 \mid m=8, y_{1}=8\right)=\frac{1}{2} \\
& \operatorname{Pr}\left(y_{3}<8 \mid m=8, y_{1}=8\right)=\frac{1}{2}
\end{aligned}
$$

and

$$
\begin{aligned}
& \operatorname{Pr}\left(y_{1}=8 \mid m=8, y_{3}<8\right)=1 \\
& \operatorname{Pr}\left(y_{3}=8 \mid m=8, y_{1}<8\right)=1
\end{aligned}
$$

Given a $l$-query $q$ and the corresponding answer $m$, the following propositions compute the probability that $y_{j}$ is equal to $m$, for each $j \in q$, the probabilistic dependencies among the sensitive values in $q$ and consider user prior-knowledge.

Proposition 1. Let $q=\left\{i_{1}, \ldots, i_{l}\right\} \subseteq K$, then, $\forall j \in q$ :

$$
\begin{gathered}
\operatorname{Pr}\left(y_{j}=m \mid \max \left\{y_{i_{1}}, \ldots, y_{i_{l}}\right\}=m\right)=\frac{2^{l-1}}{2^{l}-1} \\
\operatorname{Pr}\left(y_{j}<m \mid \max \left\{y_{i_{1}}, \ldots, y_{i_{l}}\right\}=m\right)=\frac{2^{l-1}-1}{2^{l}-1}
\end{gathered}
$$

and

$$
\begin{aligned}
& \lim _{l \rightarrow \infty} \operatorname{Pr}\left(y_{j}=m \mid \max \left\{y_{i_{1}}, \ldots, y_{i_{l}}\right\}=m\right)=\frac{1}{2} \\
& \lim _{l \rightarrow \infty} \operatorname{Pr}\left(y_{j}<m \mid \max \left\{y_{i_{1}}, \ldots, y_{i_{l}}\right\}=m\right)=\frac{1}{2}
\end{aligned}
$$

Proposition 2. Given $q=\left\{i_{1}, \ldots, i_{l}\right\}$ such that $m_{q}=$ $\max \left\{y_{i_{1}}, \ldots, y_{i_{l}}\right\}=m$, given $q^{\prime} \subset q$, with $l^{\prime}=\left|q^{\prime}\right|>0$ such 
that $m_{q^{\prime}}=\max \left\{y_{s} \mid s \in q^{\prime}\right\}=m$, then, $\forall j \in q \backslash q^{\prime}$ :

$$
\begin{aligned}
& \operatorname{Pr}\left(y_{j}=m \mid m_{q}=m, m_{q^{\prime}}=m\right)=\frac{1}{2} \\
& \operatorname{Pr}\left(y_{j}<m \mid m_{q}=m, m_{q^{\prime}}=m\right)=\frac{1}{2}
\end{aligned}
$$

EXAMPLE 8. Let $q=\{1,2,3,4,5\}$, let $m_{q}=\max \left\{y_{1}, \ldots, y_{5}\right\}$ $=m$, then if the user knows that $m_{q^{\prime}}=\max \left\{y_{4}, y_{5}\right\}=m$ then $\operatorname{Pr}\left(y_{j}=m \mid m_{q}=m, m_{q^{\prime}}=m\right)=\frac{1}{2}$, for $j=1,2,3$.

Proposition 3. Given $q=\left\{i_{1}, \ldots, i_{l}\right\}$ such that $m_{q}=$ $\max \left\{y_{i_{1}}, \ldots, y_{i_{l}}\right\}=m$, given $q^{\prime \prime} \subset q$, with $l^{\prime \prime}=\left|q^{\prime \prime}\right|>0$ such that $m_{q^{\prime \prime}}=\max \left\{y_{s} \mid s \in q^{\prime \prime}\right\}<m$, then, $\forall j \in q \backslash q^{\prime \prime}$ :

$$
\begin{array}{r}
\operatorname{Pr}\left(y_{j}=m \mid m_{q}=m, m_{q \prime \prime}<m\right)=\frac{2^{l-\left(l^{\prime \prime}+1\right)}}{2^{l-l^{\prime \prime}}-1} \\
\operatorname{Pr}\left(y_{j}<m \mid m_{q}=m, m_{q \prime \prime}<m\right)=\frac{2^{l-\left(l^{\prime \prime}+1\right)}-1}{2^{l-l^{\prime \prime}}-1}
\end{array}
$$

EXAmPle 9. Let $q=\{1,2,3,4,5\}$, if the user knows that $m_{q}=\max \left\{y_{1}, \ldots, y_{5}\right\}=m$ and $m_{q^{\prime \prime}}=\max \left\{y_{2}, y_{3}, y_{4}\right\}<m$, then $\operatorname{Pr}\left(y_{i}=m \mid m_{q}=m, m_{q \prime \prime}<m\right)=\frac{2}{3}$, for $i=1,5$. Instead, if $m_{q^{\prime \prime}}=\max \left\{y_{1}, y_{2}, y_{3}, y_{4}\right\}<m$ then $\operatorname{Pr}\left(y_{5}=\right.$ $\left.m \mid m_{q}=m, m_{q \prime \prime}<m\right)=1$

REMARK 1. In propositions 2 and $3 \max \left\{y_{s} \mid s \in q^{\prime}\right\}=m$ and respectively $\max \left\{y_{s} \mid s \in q^{\prime \prime}\right\}<m$ represent user priorknowledge. In particular if we consider $q=q_{1}$ and $q^{\prime}=q_{2}$ (resp. $q=q_{1}$ and $q^{\prime \prime}=q_{2}$ ) the equations in proposition 2 (resp. in proposition 3) represent the probabilities after the sequence of queries $\left\{q_{1}, q_{2}\right\}$.

\section{A BAYESIAN APPROACH}

In this section we present the Bayesian network as a graphical model to deal with uncertainty and show how a Bayesian network is able to deal with the on-line max auditing problem and the on-line max and min auditing problem, also in presence of duplicated sensitive values.

\subsection{Bayesian network}

A Bayesian network (BN) is a probabilistic graphical model that represents a set of variables and their probabilistic dependencies [13]. A BN, also called belief net, is a directed acyclic graph (DAG) which consists of nodes to represent variables and arcs to represent dependencies between variables. Arcs or links also represent causal influences among the variables. The strength of an influence between variables is represented by the conditional probabilities which are summarized in a conditional probability table (CPT). If there is an arc from node $A$ to another node $B, A$ is called a parent of $B$, and $B$ is a child of $A$. The set of parent nodes of a node $X_{i}$ is denoted parents $\left(X_{i}\right)$. The size of the CPT of a node $X_{i}$ depends on the number $s$ of its states, the number $n$ of parents $\left(X_{i}\right)$, and the number $s_{j}$ of parent states, in the following way:

$$
\operatorname{size}(C P T)=s \cdot \prod_{j=1}^{n} s_{j}
$$

For every possible combination of parent states, there is an entry listed in the CPT. Notice that for a large number of parents the CPT will expand drastically. A directed acyclic graph is a BN relative to a set of variables if the joint distribution of the node values can be written as the product of the local distributions of each node and its parents:

$$
\operatorname{Pr}\left(X_{1}, \ldots X_{n}\right)=\prod_{i=1}^{n} \operatorname{Pr}\left(X_{i} \mid \text { parents }\left(X_{i}\right)\right)
$$

If node $X_{i}$ has no parents, its local probability distribution is said to be unconditional, otherwise it is conditional. If the value of a node is observed, then the node is said to be an evidence node.

\subsection{Max auditing}

In this section we build a BN able to deal with on-line max auditing problem. We represent the variable $y_{i}$, for $i \in K$, and a max query, by means of a node in the BN. The model is an optimized version of the $\mathrm{BN}$ shown in [2].

Let $K=\{1,2, \ldots, n\}$ then $|P(K)|=2^{n}$. Because the number of $l$-query from the set $K$ is the binomial coefficient $C_{n}^{l}=$ $\left(\begin{array}{c}n \\ l\end{array}\right)=\frac{n !}{l !(n-l) !}$ and because we consider only $l$-query with $l>1$ then the total number of queries on $K$ is:

$$
\left(\begin{array}{c}
n \\
2
\end{array}\right)+\ldots+\left(\begin{array}{c}
n \\
n-1
\end{array}\right)+\left(\begin{array}{l}
n \\
n
\end{array}\right)=2^{n}-(1+n)
$$

Therefore, because the number of nodes grows in exponential way when $n$ increases (if we encode all max queries and variables $y_{i}$, then it needs $2^{n}-1$ nodes), we incrementally build the $\mathrm{BN}$ at run-time.

Given a $l$-query $q=\left\{i_{1}, \ldots i_{l}\right\}$, if we build the node encoding $q$ with $l$ parents $\left\{y_{i_{1}}, \ldots y_{i_{l}}\right\}$, then, by equation (7), CPT size grows exponentially, thus we decompose the $l$-ary max operator into a set of binary max operators and we build the CPTs with a minimum number of states.

We have implemented our BN model using the Hugin engine and its Java API [7].

Given a new query $q_{t}$, in order to represent the user knowledge, we consider the following phases:

1. if the node encoding the max query $q_{t}$ does not exist then we realize the temporal transformation, described in section 4.2.1, optimizing CPT size, as discussed in section 4.2 .2 ;

2. we insert evidence on node encoding the max query;

3. we check whether a probabilistic compromise occurs or not.

In this section we assume that:

- $Z=\left\{z_{1}, \ldots, z_{n}\right\}$ is a permutation of $Y=\left\{y_{1}, \ldots, y_{n}\right\}$ such that $z_{j} \geq z_{j+1}, \forall j \in\{1, \ldots, n-1\}$;

- given $S_{0}=\left\{s_{0}, s_{1}, \ldots, s_{r}, s_{r+1}\right\}$ defined in section 2.1, and a $l$-query $q=\left\{i_{1}, \ldots, i_{l}\right\}$, with $i_{j}<i_{j+1} \quad \forall j \in$ $\{1, \ldots, l-1\}$, then $\exists k \in\{1, \ldots, r\}$ such that $m=\max =$ $\left\{z_{i_{1}}, \ldots, z_{i_{l}}\right\}=z_{i_{1}}=s_{k}$ 


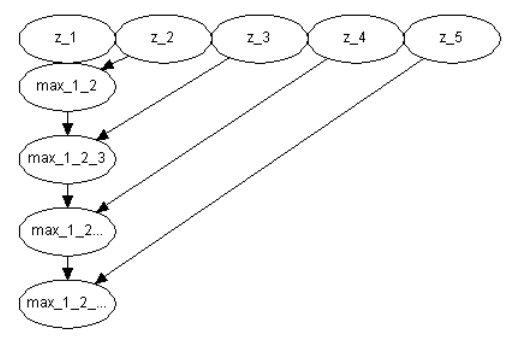

Figure 1: Temporal transformation for a max query.

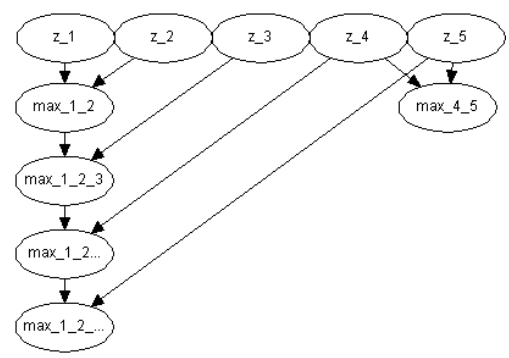

Figure 2: Sequence of temporal transformations.

- because each node encoding a $l$-query is a binary max operator, $p_{1}$ and $p_{2}$ are its parents.

\subsubsection{Decomposition of a max query}

The size of a conditional distribution that encodes the max operator can be reduced when the $l$-ary max operator is decomposed into a set of binary max operators. Two well known approaches to the decomposition are: parent divorcing [12] and temporal transformation [6].

Parent divorcing constructs a binary tree in which each node encodes the binary operator. Temporal transformation constructs a linear decomposition tree in which each node encodes the binary operator. In order to optimize our BN we use temporal transformation. For example, the BN resulting from temporal decomposition for the query $q_{1}=$ $\{1,2,3,4,5\}$ is shown in figure 1 . Each max node in the transformation has size equal to $r^{3}$, where $r$ is the domain size, in our context, $r$ is the number of distinct values of sensitive date. In the general case of a $l$-query, $l-1$ max nodes are needed. Inserting evidence on node encoding the $l$-query, we can compute the probability of the corresponding $l$ nodes in the first level.

If the auditor answers the query $\{4,5\}$, then the sequence of temporal transformations is shown in figure 2 .

Therefore, in the first level of BN there are the nodes encoding the variables $z_{j}$, and in the level $l$, with $l>1$, there are the nodes encoding the $l$-queries. We build the $\mathrm{BN}$ at run-time: given the current query $q_{t}$, we execute a temporal transformation if and only if the privacy is not breached and the answer to $q_{t}$ gives new information. If no query is answered then the BN has not nodes.

\subsubsection{Optimizing CPT size}

In this section we optimize the model by building CPT with minimum number of states.

Given $\left\{q_{1}, \ldots, q_{t-1}\right\}$ a sequence of queries already posed, $\left\{m_{1}\right.$, $\left.\ldots, m_{t-1}\right\}$ the corresponding answer and a new query $q_{t}$ with answer $m_{t}$, for each $j$ in $q_{t}$, one of the two cases is verified:

1. $\exists i \in\{1, \ldots, t-1\} \mid j \in q_{i}$ and $\mu_{j}=m_{i}$ ( $j$ is extreme element for $q_{i}$ ),

2. $\nexists$ a max query $q_{i}$ with $i \in\{1, \ldots, t-1\}, \mid j \in q_{i}$.

In the case 1 , if $\mu_{j}$, related to $\left\{q_{1}, \ldots, q_{t-1}\right\}$, is such that $\mu_{j}>m_{t}$, and in the case 2 , we set $\mu_{j}=m_{t}$.

Therefore, for each $z_{j}$ in the $\mathrm{BN}$, we have to represent the following probabilities:

$$
\begin{gathered}
\operatorname{Pr}\left(z_{j}<\mu_{j}\right) \\
\operatorname{Pr}\left(z_{j}=\mu_{j}\right) .
\end{gathered}
$$

We build the nodes in the $\mathrm{BN}$ in the following way:

NODE ENCODING $z_{j}$. The node encoding $z_{j}$ has two states $z s_{1}$ and $z s_{2}$ with $z s_{1}<z s_{2}=\mu_{j}$. Therefore, unconditional distribution table has size equal to 2 . Since we assume that the user has not knowledge about the domain of the sensitive field, the prior distribution is $\left(\frac{1}{2}, \frac{1}{2}\right)$. In our implementation we can set $z s_{1}=s_{0}$. Obviously, $z s_{2}$ is updated after a query $q_{t}$ if $\mu_{j}$, related to $\left\{q_{1}, \ldots, q_{t-1}\right\}$, is such that $\mu_{j}>m_{t}$.

NODE ENCODING A MAX QUERY. Given $q_{t}=\left\{i_{1}, \ldots, i_{l}\right\}$ the current query, $\operatorname{m}_{t}=\max \left\{z_{i_{1}}, \ldots, z_{i_{l}}\right\}=z_{i_{1}}=s_{k}, \max$ Node the node encoding the query, then maxNode has three states $m s_{1}, m s_{2}$, and $m s_{3}$, with $m s_{1}<m s_{2}=s_{k}<m s_{3}$.

Because $z_{j} \geq z_{j+1} \forall j \in\{1, \ldots, n-1\}$, also each node, in the corresponding temporal transformation, has the same states as maxNode.

Because equation (7), we have that:

- the conditional distribution table of a node encoding a 2-query has size equal to $3(2 \cdot 2)=12$;

- the conditional distribution table of a node encoding a $l$-query, with $l>2$, has size equal to $3(3 \cdot 2)=18$.

In our implementation, we set $m s_{1}=s_{0}, m s_{3}=s_{r+1}$, and define the max expression as follows:

if $\left(\operatorname{and}\left(p_{1}<s_{k}, p_{2}<s_{k}\right)\right)$ Distribution $(1,0,0)$;

else if $\left(\right.$ or $\left.\left(p_{1} \leq s_{k}, p_{2} \leq s_{k}\right)\right)$ Distribution $(0,1,0)$;

else Distribution $(0,0,1)$;

Inserting evidence on max Node in the following way:

$$
\operatorname{Pr}\left(\max N o d e=s_{k}\right)=1
$$

the $\mathrm{BN}$ is able to compute the probability in (9) and (10), for each $z_{j}$ in the $\mathrm{BN}$.

ExAmple 10. Given the table 1 , we set $s_{0}=0$, if the auditor answers $q_{1}=\{1,2,3,4,5\}$ and $q_{2}=\{4,5\}$ then the $B N$ is shown in figure 3 , and for $j \in\{1,2,3\}$, according to proposition 3,

$$
\begin{array}{r}
\operatorname{Pr}\left(z_{j}=s_{0}\right)=\operatorname{Pr}\left(z_{j}<9\right)=\frac{3}{7} \\
\operatorname{Pr}\left(z_{j}=9\right)=\frac{4}{7}
\end{array}
$$




\section{REFERENCES}

[1] N. R. Adam and J. C. Worthmann. Security-control methods for statistical databases: a comparative study. ACM Computing Surveys (CSUR), 21(4), December 1989.

[2] G. Canfora and B. Cavallo. A bayesian approach for on-line max auditing. ARES, March 2008, to appear.

[3] F. Y. Chin. Security problems on inference control for sum, max, and min queries. Journal of the ACM, 33(3):451-464, July 1986.

[4] F. Y. Chin and G. Ozsoyoglu. Auditing and inference control in statistical databases. IEEE Transaction on Software Engineering, SE-8(6):574-582, November 1982.

[5] J. Domingo-Ferrer. Inference Control in Statistical Databases: From Theory to Practice. Springer-Verlag, Berlin Heidelberg, 2002.

[6] D. Heckerman. Causal independence for knowledge acquisition and inference. pages 122-127, 1993.

[7] Hugin. www.hugin.com.

[8] K. Kenthapadi, N. Mishra, and K. Nissim. Simulatable auditing. pages 118-127. PODS, June 2005.

[9] J. Kleinberg, C. Papadimitriou, and P. Raghavan. Auditing boolean attributes. Journal of Computer and System Sciences, 66(1):244-253, February 2003.

[10] F. M. Malvestuto, M. Mezzini, and M. Moscarini. Auditing sum-queries to make a statistical database secure. ACM Transactions on Information and System Security (TISSEC), 9(1):31-60, February 2006.

[11] S. U. Nabar, B. Marthi, K. Kenthapadi, N. Mishra, and R. Motwani. Towards robustness in query auditing. pages 151-162. VLDB, September 2006.

[12] K. G. Olesen, U. Kjaerulff, F. Jensen, F. V. Jensen, B. Falck, S. Andreassen, and S. K. Andersen. A munin network for the median nerve - a case study in loops. Applied Artificial Intelligence, 3(2-3):385-403, 1989.

[13] J. Pearl. Probabilistic reasoning in intelligent systems: networks of plausible inference. Morgan Kaufmann, San Francisco, CA, USA, 1998.

[14] S. P. Reiss. Security in databases: A combinatorial study. Journal of the ACM, 26(1):45-57, January 1979. 